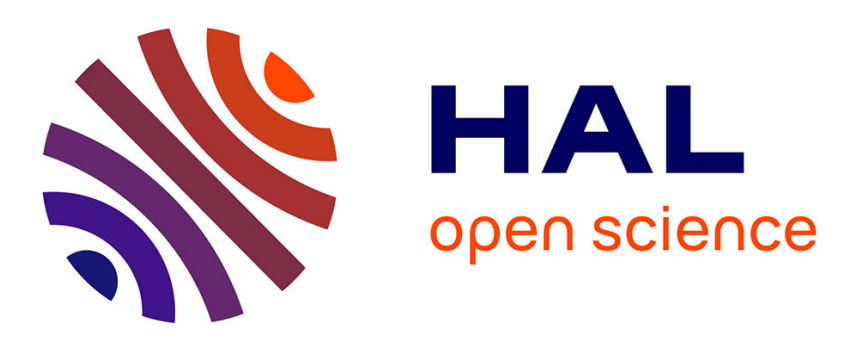

\title{
A Precise Metamodel for Open Cloud Computing Interface
}

Philippe Merle, Olivier Barais, Jean Parpaillon, Noël Plouzeau, Samir Tata

\section{To cite this version:}

Philippe Merle, Olivier Barais, Jean Parpaillon, Noël Plouzeau, Samir Tata. A Precise Metamodel for Open Cloud Computing Interface. 8th IEEE International Conference on Cloud Computing (CLOUD 2015), IEEE, Jun 2015, New York, United States. pp.852 - 859, 10.1109/CLOUD.2015.117 . hal01188800

\section{HAL Id: hal-01188800 https://hal.science/hal-01188800}

Submitted on 31 Aug 2015

HAL is a multi-disciplinary open access archive for the deposit and dissemination of scientific research documents, whether they are published or not. The documents may come from teaching and research institutions in France or abroad, or from public or private research centers.
L'archive ouverte pluridisciplinaire HAL, est destinée au dépôt et à la diffusion de documents scientifiques de niveau recherche, publiés ou non, émanant des établissements d'enseignement et de recherche français ou étrangers, des laboratoires publics ou privés. 


\section{A Precise Metamodel for Open Cloud Computing Interface}

\author{
Philippe Merle \\ Inria Lille - Nord Europe \\ Villeneuve d'Ascq, France \\ Email: philippe.merle@inria.fr
}

\author{
Olivier Barais, Jean Parpaillon, Noël Plouzeau \\ IRISA - Inria Rennes - Bretagne Atlantique \\ Rennes, France \\ Email: firstname.name@irisa.fr
}

\author{
Samir Tata \\ Institut Mines-Telecom, \\ Telecom SudParis, \\ UMR CNRS Samovar, Evry, France \\ Email: samir.tata@mines-telecom.fr
}

\begin{abstract}
Open Cloud Computing Interface (OCCI) proposes one of the first widely accepted, community-based, open standards for managing any kinds of cloud resources. But as it is specified in natural language, OCCI is imprecise, ambiguous, incomplete, and needs a precise definition of its core concepts. Indeed, the OCCI Core Model has conceptual drawbacks: an imprecise semantics of its type classification system, a nonextensible data type system for OCCI attributes, a vague and limited extension concept and the absence of a configuration concept. To tackle these issues, this paper proposes a precise metamodel for OCCI. This metamodel defines rigourously the static semantics of the OCCI core concepts, of a precise type classification system, of an extensible data type system, and of both extension and configuration concepts. This metamodel is based on the Eclipse Modeling Framework (EMF), its structure is encoded with Ecore and its static semantics is rigourously defined with Object Constraint Language (OCL). As a consequence, this metamodel provides a concrete language to precisely define and exchange OCCI models. The validation of our metamodel is done on the first world-wide dataset of OCCI extensions already published in the literature, and addressing inter-cloud networking, infrastructure, platform, application, service management, cloud monitoring, and autonomic computing domains, respectively. This validation highlights simplicity, consistency, correctness, completeness, and usefulness of the proposed metamodel.
\end{abstract}

Index Terms-Cloud Computing; Cloud Modeling; Open Cloud Computing Interface; Metamodeling;

\section{INTRODUCTION}

Cloud computing has been adopted as a dominant delivery model for computing resources [1]. This model defines three well discussed layers of services known as Infrastructure as a Service (IaaS), Platform as a Service (PaaS), and Software as a Service (SaaS) [2]. Other XaaS terms are used nowadays to name different resources provided as services in the clouds [3]. Cloud management poses different challenges [4]. Provisioning, supervising, and managing these outsourced, on-demand, pay as you go, elastic resources require cloud resource management interfaces (CRM-API). However, there is a plethora of CRM-API, proposed by Amazon, Eucalyptus, Microsoft, Google, OpenNebula, CloudStack, OpenStack, CloudBees, OpenShift, Cloud Foundry, to name a few. Even if there are several client-side API for interacting with multiple popular cloud service providers, these API are all linked to a specific programming language: Apache Libcloud for Python, Apache jclouds for Java, Gophercloud for Go, etc.
Thereby cloud computing standards are required to cope with four main issues: heterogeneity of cloud offers, interoperability between CRM-API, integration of CRM-API for building multi-cloud systems, and portability of cloud management applications. To this end, Open Cloud Computing Interface (OCCI) is an Open Grid Forum (OGF) communitybased effort to create one of the first open extensible standards for managing any kind of cloud computing resources [5].

OCCI is supported by a large community that includes providers of open source cloud software stacks such as Eucalyptus, OpenNebula, CloudStack, OpenStack, and CompatibleOne [6], and users such as the European Grid Infrastructure (EGI), to cite a few Several OCCI runtime frameworks exist, e.g., erocci, rOCCI, pySSF, pyOCNI, and OCCI4Java, and rely on Erlang, Ruby, Python, Java programming languages, respectively. OCCI has already been used successfully for managing inter-cloud networking [7], building reliable storage virtualizations [8], IaaS resources description [9], PaaS resources description [10], SaaS resources description [10], SLA negotiation and enforcement in data management [11], service management [12], resource management in federated clouds [13], cloud monitoring [14] and reconfiguration [15], and autonomic computing [16]. A common denominator of these recent usages is the use of the REST architecture style for managing cloud computing resources.

The kernel of OCCI is a generic resource-oriented metamodel called the OCCI Core Model and defined in [17]. The OCCI Core Model can be interacted with using renderings (including associated behaviours) and expanded through extensions. For instance, the OCCI HTTP Rendering [18] defines how OCCI resources are accessible as REST resources over the HTTP network protocol. The OCCI Infrastructure Extension [9] defines OCCI-compliant compute, network and storage IaaS resources.

Nevertheless, OCCI lacks a precise definition of its core concepts. Indeed, OCCI specifications are informal documents written in natural language and illustrated by UML diagrams. This informal definition of the OCCI Core Model can be interpreted in various different ways, which can lead to interoperability issues between OCCI implementations. Moreover, the OCCI Core Model has conceptual drawbacks and limita-

${ }^{1}$ http://occi-wg.org/community/implementations/ 
tions: an imprecise semantics of its built-in type classification system, a nonextensible data type system for OCCI attributes, a vague and limited extension concept, and the absence of the configuration concept.

To tackle these issues, this paper contributes a precise metamodel for OCCI. This metamodel defines rigourously the static semantics of the OCCI core concepts. Our metamodel proposes a precise type classification system, an extensible data type system, and both extension and configuration concepts. This metamodel is based on the Eclipse Modeling Framework (EMF), structured as an Ecore package and its static semantics is rigourously defined using the Object Constraint Language (OCL). As a consequence, this metamodel provides a concrete language to precisely define and exchange OCCI models. The validation of our metamodel has been done on the first worldwide dataset of OCCI extensions already published in the literature. This dataset is composed of seven OCCI extensions addressing inter-cloud networking [7], IaaS [9], PaaS [10], SaaS [10], service management [12], cloud monitoring [14], and autonomic computing [16] domains, respectively. This validation highlights simplicity, consistency, correctness, completeness, and usefulness of the proposed metamodel.

This paper is organized as follows. Section II gives background on the OCCI Core Model and identifies five conceptual drawbacks of this model. Section III describes our precise metamodel for OCCI. Section IV] validates our metamodel on seven already published OCCI extensions. Finally, Section V concludes on future perspectives.

\section{BACKGROUND AND DRAWBACKS ON THE OCCI CORE MODEL}

As illustrated by Fig. 1] the OCCI Core Model [17] is a simple resource-oriented model composed of eight concepts ${ }^{2}$

- Resource represents any cloud computing resource, e.g., a virtual machine, a network, an application container, an application. Resource owns a set of links.

- Link is a relation between two Resource instances, e.g., a computer connected to a network, an application hosted by a container. Link references to both source and target resources.

- Entity is the abstract base class of all resources and links. Each resource/link has a unique identifier, is strongly typed by a kind type and zero or more mixins types.

- Kind is the notion of class/type within OCCI, e.g., Compute, Network, Container, Application. A Kind instance owns a set of actions, can have one parent kind, and lists its entities, which are instances of this kind. There will be at least three instances of Kind: entity $\mathrm{kind}$, resource kind and link kind instances.

- Mixin is used to associate additional features, e.g., location, price, user preference, ranking, to resource/link

\footnotetext{
${ }^{2}$ Our work is based on the last revised draft of the OCCI Core Model [17], and not on the officially published and publicly available version of this document.
}

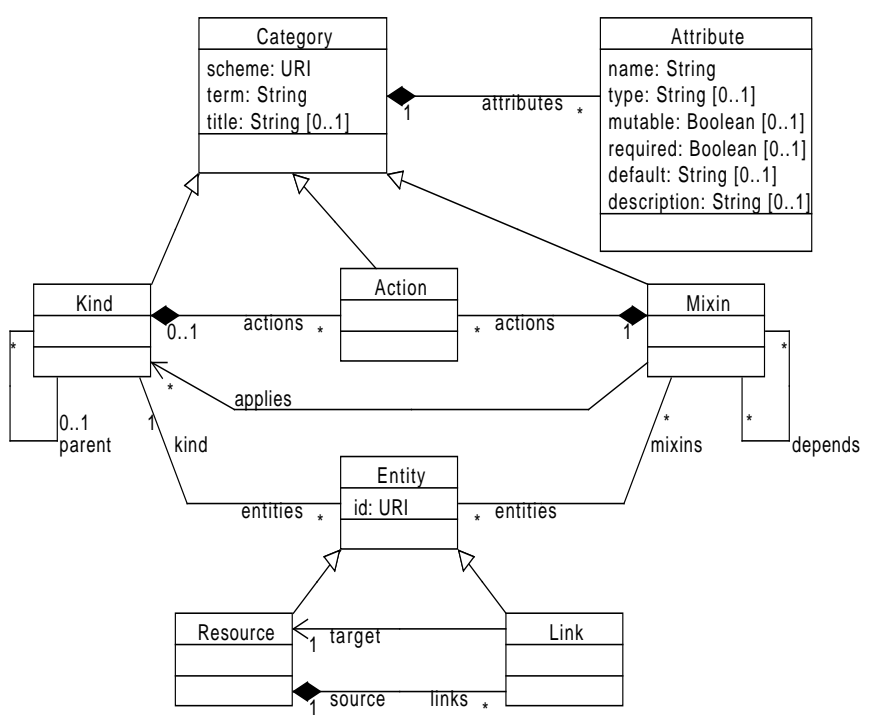

Fig. 1. UML class diagram of the OCCI Core Model (from [17])

instances. Each Mixin instance owns a set of actions, can inherit from zero or more depends mixins, defines on which kinds it can be applied, and references a set of entities on which the mixin is applied. Tagging of OCCI resource instances is supported through the association of Mixin instances (called Tags). A tag is simply a Mixin instance, which defines no additional resource capabilities, aka no attributes and no actions. Templates allow to apply predefined values to attributes of OCCI types. They are implemented using Mixin instances and they associate at entity instantiation time certain pre-populated attributes. A template is a Mixin instance with all attributes having a default value.

- Action represents an action that can be executed on entities, e.g., start a virtual machine, stop an application container, restart an application, resize a storage.

- Category is the abstract base class inherited by Kind, Mixin, and Action. Each instance of kind, mixin or action is uniquely identified by both a scheme and a term, has a human readable title, and owns a set of attributes.

- Attribute represents the definition of a client visible property, e.g., the hostname of a machine, the IP address of a network, or a parameter of an action. An attribute has one name, can have a scalar data type, can be (or not) mutable (i.e., modifiable by clients), can be (or not) required (i.e., value is provided at creation time), can have a default value and a human readable description.

Even if the OCCI Core Model is simple, it is generic enough to model any cloud computing resource such as inter-cloud networking [7], building reliable storage virtualizations [8], IaaS resources [9], PaaS resources [10], SaaS resources [10], 
service management [12], SLA negotiation and enforcement in data management [11], resource management in federated clouds [13], cloud monitoring [14] and reconfiguration [15], and autonomic computing [16]. Section [V] validates our precise metamodel for OCCI on seven of these already published works.

Through our study of OCCI in funded research projects (CompatibleOne, EASI-CLOUDS, OpenPaaS and OCCIware) we have identified five conceptual drawbacks/limitations on the OCCI Core Model:

1) Informal model: The structure of the OCCI Core Model is illustrated by the UML class diagram shown in Fig. 1. This diagram contains at least three semantic errors. In [17], Category and Entity are explicitly described as abstract types, i.e., no instance of these types can exist. Then their type name must be in italic in the UML class diagram. URI is not a base data type in UML, it must be defined. Both static and dynamics semantics of the OCCI Core Model are described by sentences and tables in natural language. Some of these sentences are imprecise and/or ambiguous and can be interpreted in various different ways, which can lead to interoperability issues between OCCI implementations. For instance, [17] does not explicitly state that two distinct attributes with the same name must not belong to the same category instance. Therefore the OCCI Core Model needs a precise and rigorous definition of its core concepts.

2) Imprecise type classification system: [17] does not explicitly state that the built-in type classification system of OCCI - parent and depends relations - must form a directed acyclic graph, i.e., a kind must not inherit from itself and a mixin must not depend from itself, both directly or transitively, else the OCCI type classification system would have a unusual semantics! A kind instance must not overload an attribute inherited from its parents directly or transitively. A mixin instance must not overload an attribute inherited from its depends directly or transitively. Entities of a mixin must have a kind compatible with an applies kind of this mixin at least. Therefore the OCCI Core Model needs a precise and rigorous definition of its type classification system.

3) Nonextensible data type system: The data type system for OCCI attributes (attribute type defined as a string) is not general enough. Currently only string, number, and boolean types are supported. This is insufficient for describing complex OCCI extensions such as OCCI Infrastructure Extension [9], as this extension uses other scalar data types like IP address, float and enumeration types. OCCI should provide some mechanisms to extend or restrict data types such as enumeration, string pattern, number range, decimal precision, etc. The OCCI data type system must be compatible with already existing and widely accepted data type systems like W3C XSD. Therefore the OCCI Core Model needs an open and extensible data type system for OCCI attributes.

4) Vague and incomplete extension concept: While the concept of extension exists in OCCI's specification, it is too vaguely and incompletely defined. Informally, each OCCI extension is a set of Kind and Mixin types targeting a concrete cloud computing domain, e.g. IaaS, PaaS, SaaS, pricing, cloud monitoring, etc. An extension can use or extend other extensions, e.g., SaaS running on PaaS deployed on IaaS implying that the SaaS extension uses the PaaS extension, which needs the IaaS extension. To make a precise and correct use of these informal use/extend dependencies in OCCI architectures the OCCI Core Model needs a precise and rigorous definition of the notion of OCCI extensions.

5) Configuration concept undefined: The concept of OCCI configurations is not explicitly defined in OCCI specifications. A configuration is an abstraction of an OCCIbased running system, and is composed of resource and link instances. A configuration must explicitly state which extensions it uses. Modeling a configuration offline could allow designers to think about and analyse their cloud systems without requiring to deploy them actually in the clouds. Therefore the OCCI Core Model needs a precise and rigorous definition of the notion of OCCI configurations.

The next section presents how these five limitations are addressed in our precise metamodel for OCCI.

\section{OCCIWARE METAMODEL}

Our precise metamodel for OCCI, named OCCIWARE Metamodel, is based on the Eclipse Modeling Framework (EMF). We have chosen EMF as this is the leading metamodeling framework, offering a plethora of metamodeling technologies such as Ecore to encode the structure of metamodels, OCL to encode the semantics of metamodels, to cite a few. The static semantics of our OCCIWARE Metamodel is rigourously defined using OCL, which is widely accepted for modeling semantics in UML and EMF, and is complete in order to help reproducibility of our contribution (see Section Availability). In the following OCL snippets are contained into simple frames and are as concise as possible to help comprehension and readability. We only use five OCL keywords: context gives the context in which an OCL snippet applies, def introduces a shortcut for an OCL expression, inv defines an invariant that must be always true, let introduces a local shortcut, and self refers to the current object on which an OCL expression is evaluated. The name of invoked OCL operations is self explained.

\section{A. Precise metamodel}

This section addresses Drawback II-1, aka OCCI's model being informal, by introducing our Ecore package and defining the static semantics of OCCI basic core concepts with five OCL invariants and four OCL definitions.

The structure of OCCIWARE METAMODEL is encoded as an Ecore package as it is illustrated in Fig. 2. Classes with a white background and their references in black encode the OCCI Core Model as defined in Section [II and illustrated by Fig. 1 The static semantics of the OCCI built-in type classification system is rigourously defined in Section III-B The EDataType class, the three String, Number, and Boolean data types, and the type reference in the orange color model our extensible data type system 


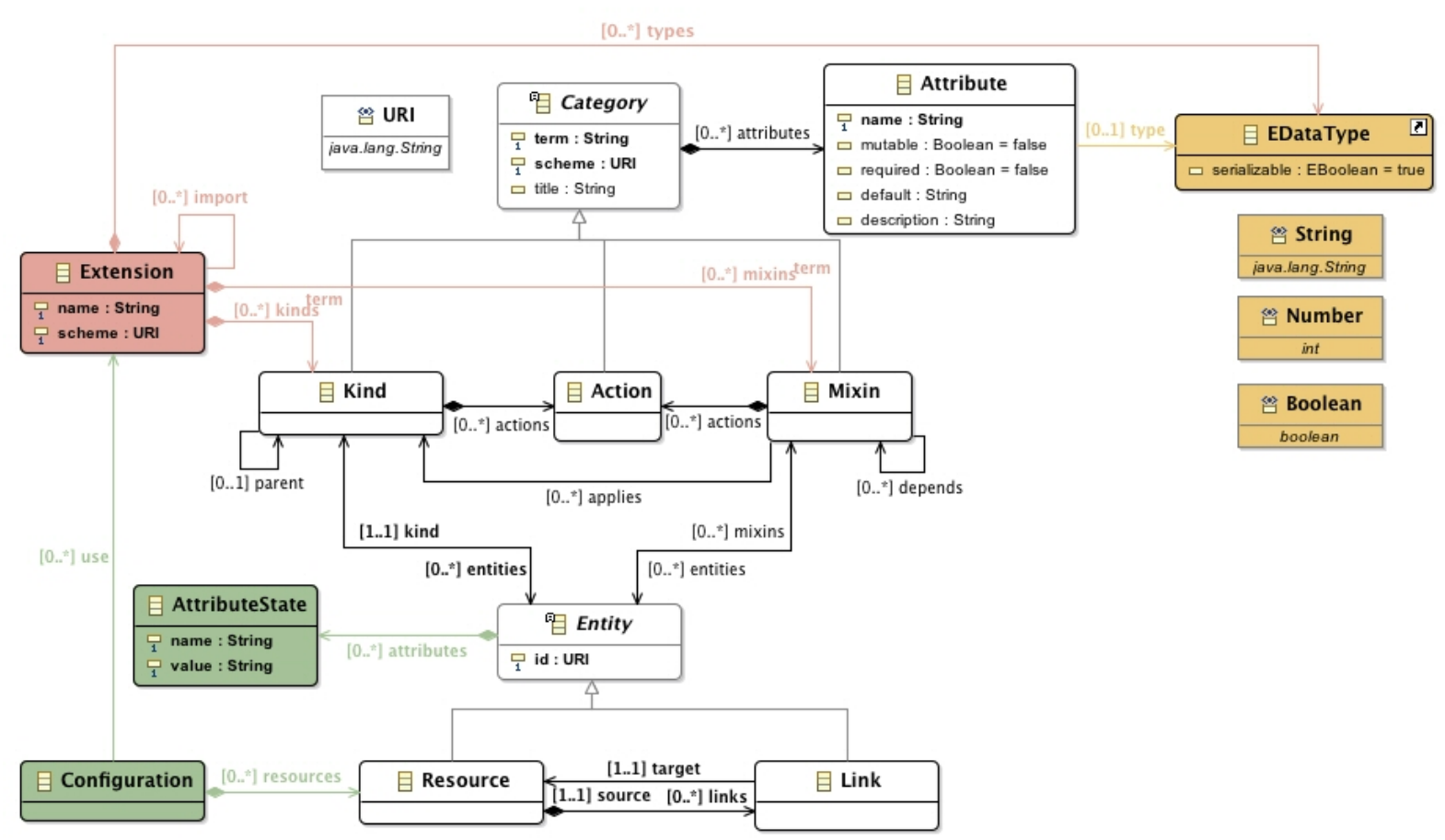

Fig. 2. Ecore diagram of OCCIWARE METAMOdEL

for OCCI attributes and are discussed in Section $\amalg I I-C$ The Extension class and its references drawn in red model the concept of OCCI extensions. Its static semantics is rigourously defined in Section III-D Both Configuration and AttributeState classes and their references drawn in green model the concept of OCCI configurations. Their static semantics is rigourously defined in Section III-E

In the following we provide basic definitions related to the OCCI Core Model: unicity of Category identity, constraints related to the Category.scheme attribute, unicity of the name of attributes of Category, Mixin tags and templates, and unicity of Entity.id.

Definition. The identity of a Category instance, aka a kind, a mixin, or an action, is equal to the concatenation of its scheme and term attributes.

context Category def: identity : String = scheme + term

Definition. Each Category instance must have a unique identity.

context Category inv: Category.allinstances () $\rightarrow$ is Unique (identity)

Definition. The scheme of each Category instance must end with a sharp.

context Category inv: scheme. substring(scheme. size() , scheme.size ()) ='\#'
Definition. The category of an Action instance is the Category instance owning this action ${ }^{3}$

context Action def: category : Category = oclContainer (). oclAsType (Category)

Definition. The scheme of an Action instance must be the concatenation of both scheme and term of its category and the suffix /action\#.

context Action inv: scheme = category. scheme. substring $(1$, category. scheme. size ()$-1)+, /,+$ category.term + / / action\#'

Definition. Each Attribute instance of a Category instance must have a distinct name.

context Category inv: attributes $\rightarrow$ is Unique (name)

Definition. A tag is a Mixin instance with no attributes and no actions. Its depends must be tags also.

context Mixin def: isTag : Boolean = attributes $\rightarrow$ isEmpty() and actions $\rightarrow$ isEmpty() and depends $\rightarrow$ for A11 (isTag)

Definition. A template is a Mixin instance with all attributes must have a default value, and all its depends mixins must be tags or templates.

${ }^{3}$ oclContainer () is a standard OCL operation returning the owner of the invoked object, here the object owning the action, and oclAsType () is a standard OCL operation to cast an object to a given class. 
context Mixin def: isTemplate: Boolean = attributes $\rightarrow$ forAll (default $\diamond$ null) and depends $\rightarrow$ forAll ( is Tag or is Template)

Definition. Each Entity instance must have a unique id.

context Entity inv: Entity. allinstances ()->isUnique( id )

\section{B. Precise type classification system}

This section addresses Drawback II-2, aka imprecise type classification system, by defining the static semantics of the OCCI built-in type classification system with eight OCL invariants.

Definition. The inheritance relation parent between Kind instances must form a direct acyclic graph. A kind instance must not inherit from itself directly or transitively

context Kind inv: parent $\rightarrow$ closure(parent) $\rightarrow$ excludes( self )

Definition. Each Kind instance must inherit from the entity $k$ ind instance directly or transitively. The entity $\mathrm{kind}$ instance is the root of the hierarchy of Kind instances.

context Kind inv: self $\rightarrow$ closure (parent) $\rightarrow$ exists $(k$ k. identity = 'http:// schemas.ogf.org/occi/core\# entity' and k.parent $=$ null)

Definition. A Kind instance must not overload an inherited attribute.

context Kind inv: attributes name->excludesAll( parent $\rightarrow$ closure (parent). attributes .name)

Definition. The inheritance relation depends between Mixin instances must form a direct acyclic graph. A mixin instance must not inherit from itself directly or transitively.

context Mixin inv: depends $\rightarrow$ closure(depends) $\rightarrow>$ excludes ( self)

Definition. A Mixin instance must not overload an inherited attribute.

context Mixin inv: attributes . name->excludesAll( depends $\rightarrow$ closure (depends). attributes . name)

Definition. The kind of an Entity instance must be compatible with one applies kind instance of each mixins of this entity.

context Entity inv: mixins $\rightarrow$ forAll $(\mathrm{m} \mid \mathrm{m}$. applies $\rightarrow$ notEmpty() implies m. applies $\rightarrow$ exists $(k \mid$ kind $\rightarrow$ closure (parent)->includes $(\mathrm{k}))$ )

${ }^{4}$ In OCL, a->closure (b) returns the set $\{$ a, a.b, a.b.b, ... $\}$.
Definition. The kind of a Resource instance must inherit from the resource $k$ ind instance directly or transitively.

context Resource inv: kind $\rightarrow$ closure(parent) $\rightarrow$ exists ( $\mathrm{k} \mid \mathrm{k}$. identity = 'http://schemas.ogf.org/occi/ core\# resource')

Definition. The kind of a Link instance must inherit from the link $\mathrm{kind}$ instance directly or transitively.

context Link inv: kind $\rightarrow$ closure (parent) $\rightarrow$ exists $(k$ $\mathrm{k}$. identity = http://schemas.ogf.org/occi/core\# link')

\section{Extensible data type system}

This section addresses Drawback II-3, aka OCCI's nonextensible data type system, by reusing the extensible data type system provided by EMF.

EMF provides an open and extensible data type system. This system is composed of two classes: EDataType to model scalar data types and EEnumType, extending EDataType, to model enumerations. An EMF data type can be restricted with metadata annotations to define regular expressions, the maximal number of digits of a number, the minimal and maximal length, minimal and maximal values, or custom constraints implemented in Java or OCL. All XSD data types are already modeled as EMF data types. Thus the EMF data type system meets all the requirements defined in Drawback II-3

In the OCCIWARE METAMODEL, the type of Attribute is modeled by the reference type from Attribute to EDataType, and the three OCCI base data types are modeled by String, Number, and Boolean data types, as shown in Fig. 2. Each OCCI extension can define its own data types.

\section{Extension concept}

This section addresses Drawback II-4, aka the vague and incomplete extension concept, by introducing the Extension class and defining the static semantics of the extension concept with six OCL invariants and two OCL definitions.

Definition. Extension represents an OCCI extension, e.g., inter-cloud networking extension [7], infrastructure extension [9], platform extension [10], application extension [10], SLA negotiation and enforcement [11], cloud monitoring extension [14], and autonomic computing extension [16]. As encoded in the Ecore package shown in Fig. 2. Extension has a name, has a scheme, owns zero or more kinds, owns zero or more mixins, owns zero or more types, and can import zero or more extensions.

Definition. Each Extension instance must have a unique scheme among all Extension instances.

context Extension inv: Extension.allinstances () $\rightarrow$ is Unique (scheme) 
Definition. The scheme of all kinds must be equal to the scheme of the owning Extension instance.

context Extension inv: kinds $\rightarrow$ forAll $(\mathrm{k} \mid \mathrm{k}$. scheme $=$ scheme)

Definition. The scheme of all mixins must start with the scheme of the owning Extension instance.

context Extension inv: mixins $\rightarrow$ forAll $(\mathrm{m} \mid \mathrm{m}$. scheme. substring $(1$, scheme. size ()$-1)=$ scheme. substring $(1$, scheme. size ()$-1))$

Definition. The extension of a Category instance is the Extension instance owning this category.

context Category def: extension: Extension = oclContainer ().oclAsType (Extension)

Definition. The following OCL function checks if a category is defined by or is imported by this extension.

context Extension def: is DefinedOrImported(category : Category) : Boolean $=1 \mathrm{et}$ e $=$ category . extension in $e=$ self or import $\rightarrow$ includes(e)

Definition. The parent of all the kinds of an extension must be defined or imported by this extension.

context Extension inv: kinds parent $\rightarrow$ forAll $(\mathrm{k}$ isDefinedOrImported $(\mathrm{k})$ )

Definition. All the depends of all the mixins of an extension must be defined or imported by this extension.

context Extension inv: mixins.depends $\rightarrow$ for All $(\mathrm{m}$ isDefinedOrImported (m))

Definition. All the applies of all the mixins of an extension must be defined or imported by this extension.

context Extension inv: mixins applies $\rightarrow$ for All (k is DefinedOrImported (k))

\section{E. Configuration concept}

This section addresses Drawback II-5, aka the absence of a configuration concept, by introducing the Configuration class and defining the static semantics of the configuration concept with six OCL invariants and one OCL definition.

Definition. Configuration represents a running OCCI system. As encoded in the Ecore package shown in Fig. 2 . Configuration owns zero or more resources (and transitively links), and use zero or more extensions. For a given configuration, the kind and mixins of all its entities (resources and links) must be defined by used extensions only. This avoids a configuration to transitively reference a type defined we do not know where.
Definition. The kind of all resources of a configuration must be defined by an extension that is explicitly used by this configuration.

context Configuration inv: use $\rightarrow$ includesAll( resources.kind. extension)

Definition. All the mixins of all resources of a configuration must be defined by an extension that is explicitly used by this configuration.

context Configuration inv: use $\rightarrow$ includesAll( resources.mixins. extension)

Definition. The kind of all links of all resources of a configuration must be defined by an extension that is explicitly used by this configuration.

context Configuration inv: use $\rightarrow$ includesAll( resources. links. kind. extension)

Definition. All the mixins of all links of all resources of a configuration must be defined by an extension that is explicitly used by this configuration.

context Configuration inv: use->includesAll( resources.links.mixins. extension)

Definition. The configuration of a Resource instance is the Configuration instance owning this resource.

context Resource def: configuration: Configuration = oclContainer ().oclAsType (Configuration)

Definition. The target resource of all links of all resources of a configuration must be a resource of this configuration.

context Configuration inv: resources. links.target $\rightarrow$ forAll $(r \mid r$.configuration = self $)$

Definition. The name of all attributes of any Entity instance must be unique.

context Entity inv: attributes $\rightarrow$ is Unique (name)

\section{F. Summary}

The OCCIware Metamodel addresses the five drawbacks identified in Section [I. This metamodel encodes all the eight core concepts of OCCI, gives a precise semantics of these concepts ( $c f$ Drawback II-1) including the type classification system ( $c f$ Drawback [II-2), reuses an extensible data type system for typing OCCI attributes ( $c f$ Drawback II-3), and introduces two new concepts: extension ( $c f$ Drawback II-4), and configuration ( $c f$ Drawback II-5). The static semantics is expressed in OCL using twenty five invariants and seven definitions. 


\section{VALIDATION}

This section presents a quantitative and qualitative evaluation of the proposed metamodel. We have set up an experimental protocol to build a dataset of already published OCCI extensions, and to analyze this dataset in respect to five qualitative criteria: Simplicity, Consistency, Correctness, Completeness, Usefulness. Subsection A details the methodology we applied to build the dataset. Subsection B analyses the dataset quantitatively and qualitatively. Subsection $\mathrm{C}$ discusses the threats to the validity of our evaluation.

\section{A. Methodology}

To evaluate our metamodel, we have surveyed the literature to find all the already published OCCI extensions. We have identified seven distinct works related to inter-cloud networking [7], infrastructure [9], [11], platform [10], application [10], service management [12], cloud monitoring [14], and autonomic computing [16] domains. As a working hypothesis, we have assumed that all these extensions are correct as they were already accepted through a peer-to-peer reviewing process. Secondly, we have encoded these seven correct extensions as instances of the OCCIWARE METAMODEL. When a work does not provide all the information required by the OCCI core model, e.g., a scheme, then we have set up this information correctly, e.g., a well-formed scheme respecting our OCL invariants. When a work is composed of several publications, e.g., autonomic computing, we have considered the last publication as the most relevant and up-to-date one. These seven encodings form what we name the OCCIWARE MODEL DATASET. To our best knowledge, this is the first world-wide dataset of OCCI extensions. Thirdly, we have passed this dataset through the EMF Validation Framework (EMF-VF), which checks all the structural constraints of our Ecore metamodel, e.g., cardinality of Ecore attributes and references, as well as all our OCL invariants. Then we have analysed our dataset and the validation results produced by EMF-VF.

\section{B. Analysis of the results}

Due to space limitations, Table I presents only a summary of our OCCIwARE Model DATASET (see Section Availability for more information). For each class of our metamodel, Table I provides the number of instances of this class present in the dataset, the number of OCL invariants defined in our metamodel, and the number of OCL invariants validated to correct by EMF-VF. The last line provides the total of OCCIWARE objects present in the dataset, of our OCL invariants, and of OCL invariants evaluated to true by EMF-VF.

This dataset covers all the five drawbacks presented in Section III and corrected by our metamodel discussed in Section III. The first eight classes are related to both informal model (II-1) and imprecise type classification system (II-2) drawbacks corrected in III-A and III-B respectively. The nonextensible data type system drawback (II-3) is addressed by the use of the EDataType class (III-C). Let's note that all the seven extensions needed to define new data types. The drawback of incomplete extension concept (II-4) was corrected by the addition of the Extension class (III-D). Finally, the configuration concept undefined drawback (III-5) was corrected by the addition of both Configuration and AttributeValue classes (III-E). Thus our dataset validates our metamodel quantitatively as all the classes and OCL constraints of our metamodel are covered by the dataset.

TABLE I

Summary of the OCCIware Model Dataset

\begin{tabular}{|c|c|c|c|}
\hline OCCIWARE & $\begin{array}{c}\text { Dataset } \\
\text { Instances }\end{array}$ & $\begin{array}{c}\text { OCCIWARE } \\
\text { Invariants }\end{array}$ & $\begin{array}{c}\text { EMF-VF } \\
\text { Validations }\end{array}$ \\
\hline Category & 123 & 3 & 369 \\
Attibute & 147 & 0 & 0 \\
Action & 56 & 1 & 56 \\
Kind & 43 & 3 & 129 \\
Mixin & 24 & 2 & 48 \\
Entity & 61 & 3 & 183 \\
Resource & 37 & 1 & 37 \\
Link & 24 & 1 & 24 \\
\hline EDataType & 42 & 0 & 0 \\
\hline Extension & 7 & 6 & 42 \\
\hline Configuration & 12 & 5 & 60 \\
AttributeValue & 157 & 0 & 0 \\
\hline Total & 549 & 25 & 948 \\
\hline
\end{tabular}

Analysing these statistics on our dataset provides an answer to the following five validation questions:

1) Simplicity: Is our metamodel simple to model cloud systems? The response to this question is yes as our metamodel contains only twelve concepts - eight from the OCCI Core Model and four new ones - allowing to model seven cloud computing domains, when other concurrent cloud standards like CIMI [19] or TOSCA [20] define a huge set of concepts, and only addressed IaaS and cloud applications, respectively.

2) Consistency: Is our precise semantics of OCCI consistent for modeling any OCCI systems? The response to this question is yes as there are no contradictions between our twenty five OCL invariants, else EMF-VF will not evaluate our whole dataset as correct (last column in Table I is equals to the number of instances multiplied by the number of invariants).

3) Correctness: Is our metamodel of OCCI correct for modeling any OCCI system? The response to this question is yes as our metamodel allows to correctly model all OCCI extensions in all observable aspects.

4) Completeness: Is our precise semantics of OCCI complete for modeling any OCCI systems? The response to this question is yes as our metamodel covers all situations encountered in the seven works proposing an OCCI extension. Moreover, our metamodel fully covers all the OCCI core concepts and addresses the five drawbacks presented into Section II.

5) Usefulness: Is our metamodel useful for modeling all OCCI systems? The response to this question is yes as our new introduced classes - Extension, EDataType, Configuration - are useful for modeling the seven OCCI extensions and fully address the five drawbacks ( $c f$ Section III. Moreover, our extensible data type system is useful in all covered extensions, i.e., new EDataType instances are created in each extension. 


\section{Threats to Validity}

Firstly, we could miss some already published OCCI extensions. These extensions could violate structural and/or OCL constraints of our OCCIWARE METAMODEL. This is a threat to validity of the correctness of our metamodel. Secondly, we could incorrectly encode some already published extensions. This could introduce a potential threat to validity on the correctness of our metamodel. We will contact the authors of these works to validate with them the correctness of our encoding of their OCCI extension. Thirdly, EMF-VF could be buggy and produce erroneous validations of incorrect OCCIware models. However, EMF-VF is in use in industry for several years so we can expect that EMF-VF is a reliable framework. Fourthly, we are not certain that we have encoded all OCL invariants covering all unexpected OCCI extensions or configurations. This is a threat to validity of the completeness of our metamodel.

\section{CONClusion And PERspectives}

OCCI proposes a generic model, APIs and protocols for managing any cloud computing resources. We argue in this paper that OCCI suffers from the absence of a precise definition of its core concepts. To address this issue, we propose a precise semantics for OCCI implemented as an Ecore metamodel with OCL invariants, and validate them on seven OCCI extensions published in the literature previously. Based on the standard OCCI Core Model [17], resource providers ambiguously described their OCCI extensions in natural language $|7|-[16]$. With our metamodel, they can now precisely encode their OCCI extensions and verify their consistency automatically.

As future work, we will continuously complete the OCCIWARE MODEL DATASET with missed or new published OCCI extensions. We will submit our metamodel to the OGF's OCCI working group gathering key world-wide OCCI specialists. We will apply our metamodel to four use cases of the OCCIware project: Datacenter as a Service, Deployment as a Service, Big Data as a Service, and Linked Data as a Service. We will define a concrete textual and graphical language to express OCCI extensions and configurations naturally. This language will be implemented in the OCCIWARE STUDIO, a model-driven tool chain providing a text editor and a graphical modeler to think about, design, model, and analyse OCCI extensions and configurations easily. Model-based generators will help to generate artefacts such as various forms of documentation as well as executable code for existing OCCI runtime frameworks, e.g. as erocci, OCCI4Java, rOCCI, pySSF, pyOCNI, etc. Finally, we will define an execution semantics of OCCI, i.e. an operational semantics for Create, Read, Update, Delete (CRUD) operations of OCCI and a behaviour semantics for OCCI actions. This will make OCCIWARE models executable natively inside a Models@run.time interpreter framework.

\section{AVAILABILITY}

Readers can find both our precise OCCIware Metamodel (the Ecore package and all OCL invariants) and OCCIware Model Dataset at the following address: https:/github.com/occiware/ecore/tree/master/metamodel/

\section{ACKNOWLEDGMENT}

This work is supported by the OCCIware (www.occiware. org) research and development project funded by French Programme d'Investissements d'Avenir (PIA).

\section{REFERENCES}

[1] M. Armbrust, A. Fox, R. Griffith, A. D. Joseph, R. Katz, A. Konwinski, G. Lee, D. Patterson, A. Rabkin, I. Stoica et al., "A View of Cloud Computing," Communications of the ACM, vol. 53, no. 4, pp. 50-58, 2010.

[2] P. Mell and T. Grance, "The NIST Definition of Cloud Computing," NIST Special Publication, vol. 800, no. 145, Sep. 2011.

[3] P. Banerjee, C. Bash, R. Friedrich, P. Goldsack, B. A. Huberman, J. Manley, C. Patel, P. Ranganathan, and A. Veitch, "Everything as a Service: Powering the new information economy," IEEE Computer, vol. 44, no. 3, pp. 36-43, 2011.

[4] J. Martin-Flatin, "Challenges in Cloud Management," IEEE Cloud Computing, vol. 1, no. 1, pp. 66-70, 2014

[5] A. Edmonds, T. Metsch, A. Papaspyrou, and A. Richardson, "Toward an Open Cloud Standard," IEEE Internet Computing, vol. 16, no. 4, pp. $15-25,2012$.

[6] S. Yangui, I.-J. Marshall, J.-P. Laisne, and S. Tata, "CompatibleOne: The Open Source Cloud Broker," Journal of Grid Computing, vol. 12, no. 1, pp. 1-17, 2013.

[7] H. Medhioub, B. Msekni, and D. Zeghlache, "OCNI - Open Cloud Networking Interface," in 22nd International Conference on Computer Communications and Networks (ICCCN). IEEE, 2013, pp. 1-8.

[8] B. Nagarajan and J. Suguna, "A Review on Cloud Data Storage in Virtual Perspective," International Journal of Computer Science and Information Technologies, vol. 5, no. 5, 2014.

[9] T. Metsch and A. Edmonds, "Open Cloud Computing Interface Infrastructure," Open Grid Forum, OCCI-WG, Specification Document GFD-P-R.184, Oct. 2010.

[10] S. Yangui and S. Tata, "An OCCI Compliant Model for PaaS Resources Description and Provisioning," The Computer Journal, 2014, in press. [Online]. Available: http://comjnl.oxfordjournals.org/content/early/2014/ 11/19/comjnl.bxu132.abstract

[11] A. Edmonds, T. Metsch, and A. Papaspyrou, "Open Cloud Computing Interface in Data Management-Related Setups," in Grid and Cloud Database Management, S. Fiore and G. Aloisio, Eds. Springer, 2011, pp. 23-48.

[12] A. Ghrab, S. Skhiri, H. Kœner, and G. Leduc, "Towards a standardsbased cloud service manager," in 3rd International Conference on Cloud Computing and Services Science (CLOSER 2013), 2013.

[13] M. Mosch, S. Groß, and A. Schill, "User-controlled resource management in federated clouds," Journal of Cloud Computing, vol. 3, no. 1, pp. 1-18, 2014.

[14] A. Ciuffoletti, "A Simple and Generic Interface for a Cloud Monitoring Service," in 4th International Conference on Cloud Computing and Services Science (CLOSER 2014), Apr. 2014, pp. 143-150.

[15] M. Mohamed, D. Belaïd, and S. Tata, "Monitoring and Reconfiguration for OCCI Resources," in 5th IEEE International Conference on Cloud Computing Technology and Science (CloudCom 2013), vol. 1, Dec. 2013, pp. 539-546.

[16] M. Mohamed, M. Amziani, D. Belaid, S. Tata, and T. Melliti, "An autonomic approach to manage elasticity of business processes in the cloud," Future Generation Computer Systems, Available online 22 October 2014. [Online]. Available: http://www.sciencedirect.com/ science/article/pii/S0167739X14002106

[17] R. Nyrén, A. Edmonds, A. Papaspyrou, and T. Metsch, "Open Cloud Computing Interface - Core," Open Grid Forum, OCCI-WG, Specification Document GFD-P-R.183, Apr. 2011, errata update available at http: //redmine.ogf.org/projects/occi-wg/repository/show?rev=core-errata

[18] T. Metsch and A. Edmonds, "Open Cloud Computing Interface - HTTP Rendering," Open Grid Forum, OCCI-WG, Specification Document GFD-P-R.185, Apr. 2011

[19] D. Davis and G. Pilz, "Cloud Infrastructure Management Interface (CIMI) Model and REST Interface over HTTP," vol. DSP-0263, May 2012.

[20] T. Binz, U. Breitenbücher, F. Haupt, O. Kopp, F. Leymann, A. Nowak, and S. Wagner, "OpenTOSCA - A Runtime for TOSCA-based Cloud Applications," in Service-Oriented Computing. Springer, 2013, pp. 692695. 